\title{
Impact of the time of diagnosis on the postoperative outcome of newborn infants with congenital heart disease in a public hospital in Argentina
}

\author{
Mariela Krynski, M.D., ${ }^{a}$ Mercedes Montonati, M.D., ${ }^{a}$ and MaríaAlthabe, M.D. ${ }^{a}$
}

\begin{abstract}
Congenital heart diseases account for $13 \%$ of child mortality, and late diagnosis increases morbidity and mortality. The objective of this study was to assess the impact of the time of diagnosis in newborn infants on the postoperative course. The time of diagnosis was classified into prenatal, before or after discharge from the maternity center. Two hundred ninety-nine patients were included; their gestational age was $38 \pm 2.6$ weeks and their birth weight was $3.22 \pm 0.6 \mathrm{~kg}$. Twohundred sixty-six patients underwentsurgery, 13 were excluded due to the characteristics of the lesion, and 10 because of hemodynamic collapse, while 10 were treated at the Cath Lab or were operated beyond the neonatal period. Only 19 patients $(7 \%)$ were diagnosed before birth; most were patients who had health insurance, lived in the City of Buenos Aires or in capital cities of other provinces. The time of diagnosis was not associated with differences in mortality; however, an association was observed with a poor preoperative clinical status, with $3.6 \%$ of patients who died before surgery. Postoperative survival was $89.5 \%$; overall survival was $83 \%$. Key words: congenital heart disease, heart surgery, prenatal diagnosis
\end{abstract}

http://dx.doi.org/10.5546/aap.2015.eng.433

\section{INTRODUCTION}

Congenital heart diseases (CHDs) are the first cause of death among patients with congenital malformations. CHDs affect 8-12/1000 live newborn infants and are some of the most common and potentially severe congenital malformations. In Argentina, CHDs account for $13 \%$ of child mortality, and are the third

a. Intensive Care Unit 35 - Cardiovascular Recovery Unit. Hospital de Pediatría "Prof. Dr. Juan P. Garrahan".

Autonomous City of Buenos Aires, Argentina.

E-mail Address:

Mariela Krynski, M.D.: marikrynski@hotmail.com

Funding: None.

Conflict of Interest: None.

Received: 12-16-2014

Accepted: 5-4-2015 leading cause of death following prematurity and infections. ${ }^{1}$ Many times, a late diagnosis results in a high perioperative morbidity and mortality. In this regard, different strategies have been developed to improve the diagnosis of congenital heart diseases. These include prenatal diagnosis, which allows to schedule delivery at or near a specialized medical center, and anticipating what specific support the infant will need at birth, such as prostaglandins, septostomy or emergency surgery. Routine pulse oximetry for newborn infants, as a screening strategy, may be useful to improve the diagnostic opportunity among these patients. ${ }^{2,3}$ In our setting, there are no studies providing information on the time of diagnosis and its potential impact on treatment. The objective of this study is to describe the characteristics of newborn infants with CHDs admitted to the Intensive Care Unit of Hospital Garrahan (UCI 35) and assess the impact of the time of diagnosis on postoperative outcomes.

\section{PATIENTS AND METHODS}

All patients younger than 60 days admitted to the ICU 35 and diagnosed with a CHD that could be surgically treated in the neonatal period between 2007 and 2011 were included. The following demographic data were recorded: age at the time of diagnosis, sex, weight, place of residence, antenatal care, health insurance, and family history of heart disease. Diagnostic information: time of diagnosis, type of heart disease, main symptom, clinical status, and diagnostic consistency. Data on surgery and postoperative period: type of surgical repair and risk-adjusted mortality with RACHS-1 score. An age limit of 60 days old was established to include patients with a late diagnosis of CHD who could be managed routinely in the neonatal period. The time of diagnosis was classified into prenatal, before or after discharge from the maternity unit. Symptoms were recorded as cyanosis, shock, murmur, and heart failure. Clinical status was considered poor if the patient required mechanical ventilation and/or intravenous inotropic agents upon admission to 
the ICU. Diagnosis was considered consistent if the referral diagnosis matched the patient's major defect, even if other minor associated defects had been identified. The type of surgical repair was grouped by complexity as per the RACHS-1 scale, which consists of six mortality risk categories in ascending order according to the complexity of the surgical procedure. ${ }^{4} \mathrm{~A}$ descriptive statistical analysis was performed; data were reported as mean and standard deviation (SD) or as percentage, as applicable. The time of diagnosis and the clinical status upon admission were assessed as risk factors for mortality (Stata 9.0). The protocol was approved by the hospital's research committee (No. 849/2014). This was a retrospective study without intervention, so it was not necessary to request an informed consent.

\section{RESULTS}

Two hundred ninety-nine patients were included; their average gestational age was $38 \pm 2.6$ weeks and their birth weight was $3.22 \pm 0.6 \mathrm{~kg}$. Out of the total population, 266 patients underwent surgery, 13 were excluded due to the characteristics of lesions or severe associated malformations (12 had a single ventricle), and 10 due to hemodynamic collapse, while 10 were treated at the Cath Lab or had surgery beyond the neonatal period (Figure 1). In the studied population, $11 \%$ did not have comprehensive antenatal care, $63 \%$ were born by vaginal delivery; only $2.3 \%$ had a history of siblings with congenital heart disease, $19 \%$ had health insurance coverage. In relation to the place of residence, $47 \%$ were from the province of Buenos Aires, 53\% from other provinces, and $7 \%$ lived in the Autonomous City of Buenos Aires (CABA). Only 19 patients $(7 \%)$ received a prenatal diagnosis, $65 \%$ were diagnosed during hospitalization, and $28 \%$ at the time of discharge from the maternity center. Among those patients with prenatal diagnosis, 10 had different types of single ventricle defects (Table 1). Patients who had health insurance had better chances of being diagnosed in the prenatal period $(12.5 \%$ vs. $5.1 \%$, $p=0.047$ ).

Likewise, those living in CABA or in other capital cities had better chances of having a prenatal diagnosis than those living in other provincial areas $(8.7 \%$ versus $1.2 \%, p=0.019)$. The most frequent clinical presentation detected was cyanosis in $68 \%$ of cases, regardless of the time of diagnosis, followed by murmur in $15 \%$, shock in $9 \%$, congestive heart failure (CHF) in $8 \%$. Fiftythree percent of patients were on mechanical ventilation and receiving IV inotropic agents and/or prostaglandins upon admission to the ICU. Considering the entire population, the most common type of CHD was simple and complex transposition of the great arteries (TGA); the rest of the diagnoses are described in Table 2. Diagnosis was confirmed by color Doppler scanning performed upon admission. Consistency with the diagnosis made at the referral facility was $78 \%$. Seventy-eight patients $(26 \%)$ required additional procedures, such as catheterization and/or a computerized axial tomography (CAT) scan. Of all patients with an indication for surgery (266), 3.4\% were not operated because of their

FIGURE 1. Indications for intervention in the total of the population

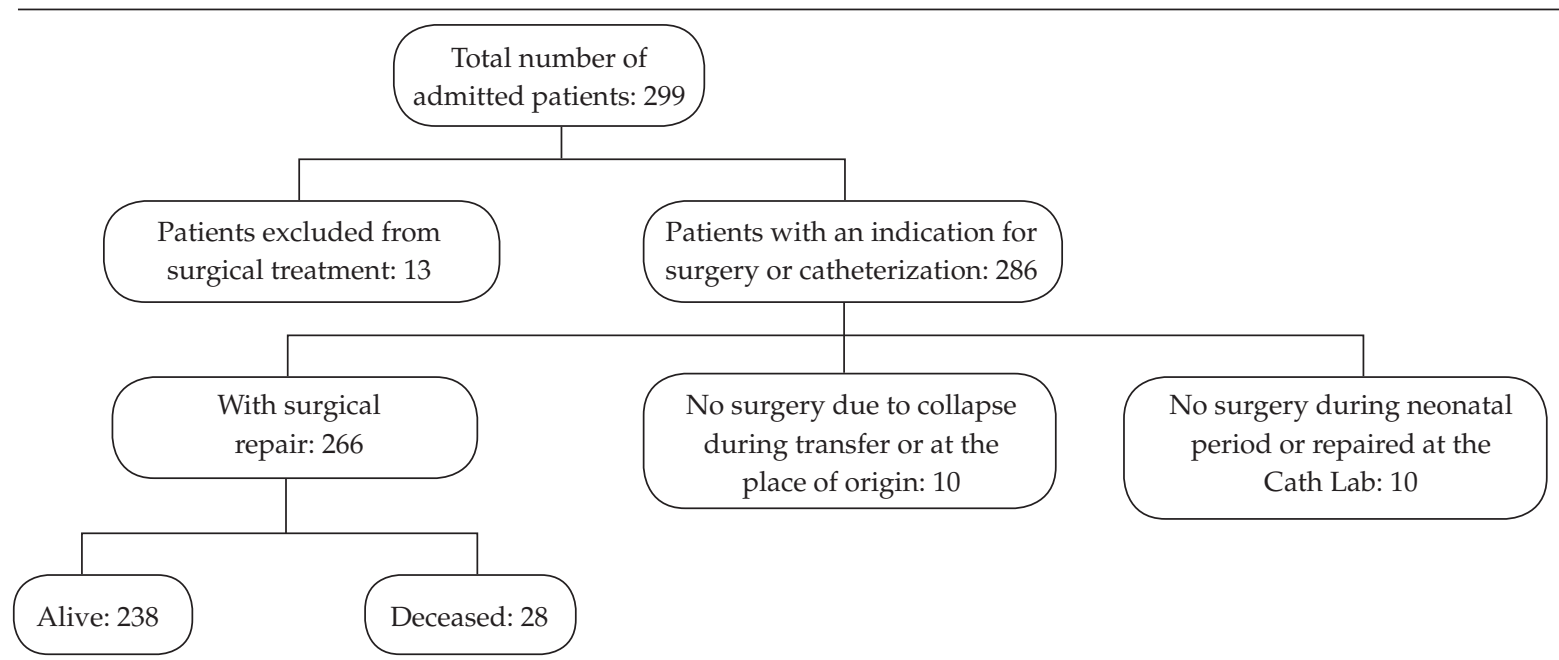


poor clinical status and died; most of them had a chance of undergoing a good anatomical repair (three had TGA, two had interruption of the aortic arch with ventricular septal defect [VSD], and one had truncus arteriosus). As per the RACHS-1 scale, surgeries were highly complex, and more than $70 \%$ of cases were in the $\geq 3$ category (R1: 1\%; R2: 15\%; R3: 40\%; R4: 31\%; and R5/6: 13\%). Postoperative mortality was $10.5 \%$, while overall mortality in this series reached $17 \%$. In the univariate analysis, the time of diagnosis was not significantly associated with differences in mortality $(18 \%$ with prenatal diagnosis versus $9.7 \%$ with postnatal diagnosis, $p=0.25$ ). However, mortality was higher among patients with a poor previous status $(22 \%$ versus $9 \%, p=0.003)$.

TABLE 1. Malformations with prenatal diagnosis

\begin{tabular}{lc}
\hline Condition & Number \\
\hline LV hypoplasia & 9 \\
Pulmonary atresia with VSD & 3 \\
Total anomalous pulmonary venous return & 2 \\
VSD with coarctation of the aorta & 2 \\
Transposition of the great arteries & 1 \\
Single ventricle without LV hypoplasia & 1 \\
Truncus arteriosus & 1 \\
Total & 19 \\
\hline
\end{tabular}

VSD: ventricular septal defect; LV: left ventricle.

\section{DISCUSSION}

Advances made in neonatal cardiovascular surgery, surgical techniques, anesthesia and perfusion have led to major improvements in survival of patients with highly-complex malformations. An early diagnosis is one of the bases of the timely management of these patients because it allows to achieve an adequate preoperative stabilization, a suitable coordination to transfer patients, and better preoperative conditions. ${ }^{5}$ As seen in our series, patients in a poor preoperative condition have a worse surgical outcome and may even miss the chance to undergo repair surgery. ${ }^{6}$ Although diagnosis has improved in the past years, many patients are still diagnosed after hospital discharge and the percentage of patients with prenatal diagnosis is very low. Patients born in CABA or province of Buenos Aires and/or those who had health insurance coverage had better chances of being diagnosed prenatally, thus evidencing certain inequalities in the health system. This is particularly noteworthy because it is a selected population given that Hospital Garrahan is the largest referral tertiary care facility specialized in pediatric cardiovascular surgery in Argentina, where 60 newborn infants undergo surgery with cardiopulmonary bypass every year. In the literature, similar facilities describe $30-50 \%$ of this type of patients with prenatal diagnosis. ${ }^{78}$ Prenatal diagnosis itself is not related to a lower postoperative mortality,

TABLE 2. Diagnosis by order of frequency

\begin{tabular}{lcc}
\hline Congenital heart disease & Absolute frequency & Relative frequency (\%) \\
\hline TGA & 82 & 27.4 \\
LVH & 41 & 13.7 \\
TAPVR & 40 & 13.4 \\
SV without LVH & 28 & 9.3 \\
VSD + CoA & 18 & 6.0 \\
PA with VSD & 13 & 4.3 \\
IAA & 13 & 4.3 \\
CoA & 12 & 4.0 \\
Truncus & 10 & 3.3 \\
VSD & 7 & 2.3 \\
Fallot & 4 & 1.3 \\
Shone & 2 & 0.6 \\
Atrioventricular canal & 2 & 0.6 \\
Ductus & 1 & 0.3 \\
Others & 26 & 8.7 \\
Total & 299 & \\
\hline
\end{tabular}

TGA: transposition of the great arteries; LVH: left ventricle hypoplasia; TAPVR: total anomalous pulmonary venous return; SV without LVH: single ventricle without left ventricle hypoplasia; VSD + CoA: ventricular septal defect + coarctation of the aorta; PA with VSD: pulmonary atresia with ventricular septal defect; IAA: interruption of the aortic arch. 
since an unbelievable number of variables have an impact on such outcome, for example, the different number of conditions and surgical procedures involved. However, it is very important to reduce congenital heart disease mortality in such a large country with a few healthcare facilities specialized in the treatment of such complex cases and where it is not easy to comply with referral and transfer procedures. ${ }^{9}$ Postoperative mortality with neonatal heart surgery was overall $10.5 \%$, while mortality related to these conditions when not managed surgically reached $90 \%$. It is therefore clear that reducing mortality related to congenital heart disease can be achieved through repair or palliative surgery provided malformation characteristics allow this approach. In our series, if the ten patients who missed their chance for surgery had undergone the operation, overall mortality might have been reduced by $3 \%$, from $17 \%$ to $14 \%$. Delays in postnatal diagnosis are related to several causes: lack of suspicion, lack of trained healthcare providers, or it might be related to patients with fewer symptoms. These may be patients with less severe presentations or whose symptoms are related to hemodynamic changes occurred in the neonatal period, such as ductus closure or reduction in pulmonary resistance. Based on the design of this study, it is not possible to draw conclusions in this regard or estimate the number of patients with severe malformations who may have died without being diagnosed with a congenital heart disease. For these reasons, we believe that when no prenatal diagnosis is made, a pulse oximetry screening at the time of discharge may be a useful strategy to improve the diagnostic opportunity and reduce morbidity and mortality related to congenital heart diseases. ${ }^{2}$ Although in this study no other long-term impact outcome was assessed, such as neurological damage, the literature focuses on reducing early comorbidities, such as preoperative seizures and asphyxia, which have long-term sequelae on the neurological development, and on those heart conditions that appear in the first days of life, such as shock. ${ }^{10-12}$ Diagnostic consistency with the referral facilitymay be considered reasonable, since $26 \%$ of cases required additional procedures thereby reflecting the complexity of this group of conditions. Such information is relevant to establish the level of training required for this type of diagnosis.

\section{CONCLUSIONS}

Congenital heart disease diagnosis was made in the prenatal period in $7 \%$ of cases, and after discharge in $28 \%$. Living in the metropolitan area and/or having health insurance were associated with a better chance of receiving a prenatal diagnosis. The time of diagnosis was not associated with a higher postoperative mortality; however, $3.6 \%$ of patients with an indication for surgical repair died before being subjected to the procedure due to their poor clinical status. Survival of patients who underwent surgery was $89.5 \%$.

\section{REFERENCES}

1. Argentina. Ministerio de Salud. Dirección de Estadísticas e Información en Salud. Estadísticas vitales. Información básica año 2012.BuenosAires,2013. [Accessed on: October 10, 2014]. Available at: http:/ / www.deis.gov.ar / publicaciones/archivos/Serie5Nro56.pdf.

2. Mahle WT, Martin GR, Beekman RH $3^{\text {rd }}$, Morrow WR. Endorsement of Health and Human Services recommendation for pulse oximetry screening for critical congenital heart disease. Pediatrics 2012;129(1):190-2.

3. Peterson C, Ailes E, Riehle-Colarusso T, Oster ME, et al. Late detection of critical congenital heart disease among US infants: estimation of the potential impact of proposed universal screening using pulse oximetry. JAMA Pediatr 2014;168(4):361-70.

4. Jenkins KJ,GauvreauK, NewburgerJW,Spray TL, etal.Consensus-based method for risk adjustment for surgery forcongenital heart disease.JThoracCardiovascSurg2002;123(1):110-8.

5. Dawson AL, Cassell CH, Riehle-Colarusso T, Grosse SD, et al. Factors associated with late detection of critical congenital heart disease in newborns. Pediatrics 2013; 132(3):e604-11.

6. Chang RK, Gurvitz M, Rodriguez S. Missed diagnosis of critical congenital heart disease. Arch Pediatr Adolesc Med 2008;162(10):969-74

7. Fuchs IB, Müller H, Abdul-Khaliq H, Harder T, et al. Immediate and long-term out comes in children with prenatal diagnosis of selected isolated congenital heart defects. Ultrasound ObstetGynecol2007;29(1):38-43.

8. Brown KL, Ridout DA, HoskoteA, Verhulst L, et al. Delayed diagnosis of congenital heart disease worsens preoperative condition and out come of surgery in neonates.Heart 2006;92(9):1298-302.

9. SatomiG, YasukochiS,Shimizu T, TakigikuK, etal. Has fetal echocardiography improved the prognosis of congenital heart disease? Comparison of patients with hypoplastic left heart syndrome with and without prenatal diagnosis. Pediatr Int1999;41(6):728-32.

10. Mahle WT, Clancy RR, McGaurn SP, Goin JE, et al. Impact of prenatal diagnosis on survival and early neurologic morbidity in neonates with the hypoplastic left heart syndrome. Pediatrics 2001;107(6):1277-82.

11. Bartlett JM, Wypij D, Bellinger DC, Rappaport LA, et al. Effect of prenatal diagnosis on outcomes in D-transposition of the great arteries. Pediatrics 2004;113(4):e335-40.

12. Kumar RK, Newburger JW, Gauvreau K, Kamenir SA, etal.Comparison of out come when hypoplastic left heart syndrome and transposition of the great arteries are diagnosed prenatally versus when diagnosis of these two conditions is made only postnatally. Am J Cardiol 1999;83(12):1649-53. 